\title{
UN VIAJE AL INTERIOR DE LA LECTURA CON JULIO VERNE
}

\author{
Ma Begoña Carretero Gómez \\ I.E.S. Isabel la Católica. Guadahortuna (Granada). \\ begocarretero@hotmail.com
}

[Recibido en Enero de 2008, aceptado en Abril de 2008]

\begin{abstract}
RESUMEN(Inglés)
Este trabajo se ha centrado en la lectura de textos de ciencia ficción en las clases de ciencias. El objetivo más importante de esta experiencia ha sido conseguir que los alumnos desarrollen la capacidad de relacionar conceptos que aparecen en la lectura con los conocimientos adquiridos previamente en clase. Como bien dice Loscertales y Núñez (1997) los alumnos de nuestro sistema educativo son los que configuran el trabajo del profesor, el cual deberá poner en práctica todos los recursos a su alcance para adecuar el proceso de enseñanza-aprendizaje a los intereses, necesidades, y características del alumnado. Por ello la idea de acudir a la lectura de textos de ciencia ficción sería una buena forma de hacer este proceso más atractivo. Es muy importante acercar la ciencia y hacerla más accesible. La ciencia ficción ayuda a fomentar el interés por las ciencias estimulando la curiosidad del alumnado, por lo que utilizarla en las clases Biología ha sido una buena estrategia para conseguir aproximar la ciencia y mejorar la capacidad lectora del alumnado. Para llevarla a cabo se ha elegido la novela "Viaje al centro de la Tierra" de Jules Verne.
\end{abstract}

Palabras clave: Enseñanza de las ciencias; literatura de ciencia ficción; fomento de la lectura; recurso didáctico; métodos de la ciencia; alfabetización científica.

\section{INTRODUCCIÓN}

Uno de los elementos esenciales de la cultura de nuestros días es la educación científica. Es imprescindible dotar a la ciudadanía de una cultura científica suficiente que le permita desenvolverse en el mundo actual. Sin embargo es posible detectar como en el ámbito escolar aparece cierto grado de fracaso unido con una visión deformada de la ciencia, así como de un menor interés por parte del alumnado a todo lo que conlleva la ciencia y su aprendizaje. Si a todo ello le unimos la necesidad de mejorar la comprensión lectora, hay motivos suficientes para intentar mejorar las habilidades de los ciudadanos del futuro. Hay que tener en cuenta que la ciudadanía en general posee una imagen diferente de la ciencia y de los científicos, que en muchas ocasiones son transmitidas por los diferentes medios de comunicación: cómics, novelas, cine, etc. (Gallego, 2007). 


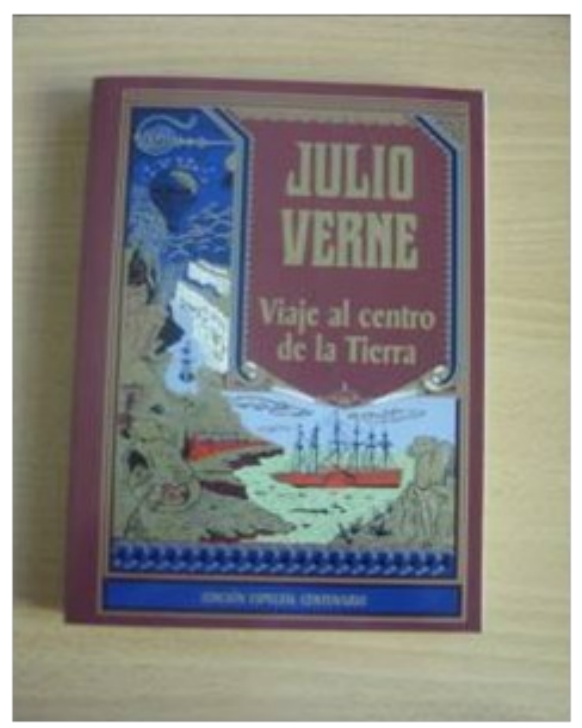

Este trabajo se ha centrado en la lectura de textos de ciencia ficción en las clases de ciencias, con la doble finalidad de: desarrollar la capacidad lectora del alumnado $y$, por otro difundir la ciencia de una forma más amena y accesible, contribuyendo así a la alfabetización científica de los estudiantes. Es necesario promover la enseñanza de estrategias variadas de comprensión lectora en las clases de ciencias utilizando textos de diferentes características (Maturano, Mazzitelli y Macías 2006). El correcto manejo del lenguaje es una herramienta básica para el aprendizaje de manera que el profesorado de ciencias debe contribuir a ello desde su asignatura (Márquez y Prat, 2005), actuando como puente que relacione todas las actividades realizadas en el aula y facilitando a los alumnos la construcción de su propio conocimiento. El problema surge al encontrar parte del alumnado con dificultades en lectura y escritura, no comprenden lo que leen, hay casos en los que su lectura no es fluida si no que "silabean". Esto ha hecho que el claustro de profesores del centro decidiera llevar a cabo un plan de lectura para el instituto. Desde el departamento de ciencias naturales, y en concreto desde la asignatura de Biología y Geología, se ha tratado colaborar en el mismo poniendo en marcha esta experiencia.

La lectura fluida y comprensiva es una herramienta fundamental para el aprendizaje de cualquier asignatura, además no solo es uno de los recursos más utilizados durante la vida escolar, sino que constituye uno de los instrumentos fundamentales para poder seguir aprendiendo el resto de nuestra vida (Márquez y Prat 2005). Hay que perseguir que el alumnado que termina la escolaridad obligatoria siga leyendo e informándose por el simple hecho de aprender y saciar su curiosidad con la lectura.

Sin embargo los profesores de ciencias naturales no solo nos encontramos con el problema de la deficiencia lectora del alumnado, sino que por otra parte en una sociedad en la que la ciencia y la tecnología son cada vez más importantes en nuestras vidas, podemos observar como es insuficiente el interés por estas disciplinas consideradas para "elegidos". A menudo se insiste en la idea de que el trabajo científico es algo reservado para minorías especialmente dotadas, donde los conocimientos científicos aparecen como obra de genios aislados, ignorándose el trabajo en equipo, con claras discriminaciones de carácter social y de género (Fernández, Gil, Carrascosa, Cachapuz y Praia 2002). Este desinterés existente en la sociedad en general, se refleja diariamente en las aulas lo que origina un reto añadido: trabajar con recursos que sean más atractivos que las simples actividades del libro

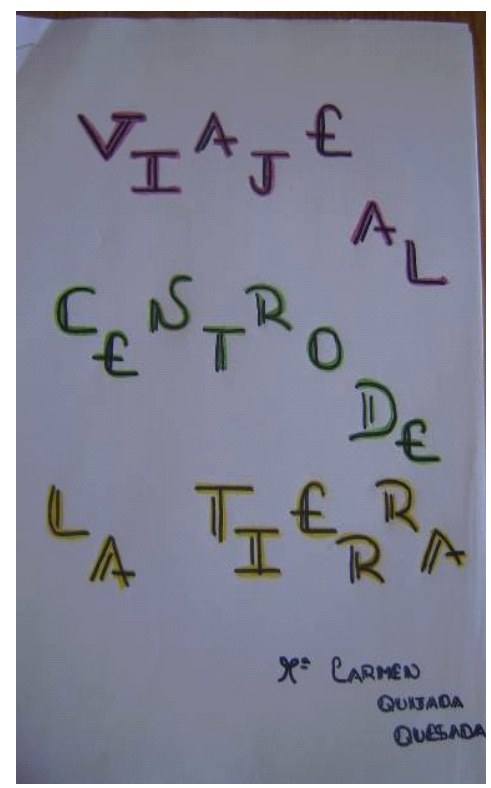


de texto (Guerra, 2005) o del simple "cacharreo" de laboratorio. Los alumnos ya empiezan a estar cansados de documentales, videos, Internet, enciclopedias, etc. Así que el uso la literatura de ciencia ficción podría aportar un "granito de arena".

\section{JUSTIFICACIÓN: QUE RAZONES NOS MOVIERON A REALIZAR ESTE "VIAJE"}

Cuando comencé mi labor docente llegue a ella con la idea equivocada de que el alumnado al que me enfrentaba, manejaba perfectamente la comprensión lectora. Poco a poco empezaron a llamarme la atención la gran cantidad de faltas de ortografía que encontraba al corregir exámenes y trabajos. Mi asombro fue mayor cuando decidí que parte del tiempo de clase lo dedicáramos a la lectura en voz alta y descubrí las deficiencias que presentaban. Tenía ciertas sospechas acerca de su dificultad lectora puesto que en ocasiones observaba que las respuestas dadas no eran acordes con lo que se les preguntaba, efectivamente al leer en voz alta salieron a la luz numerosas carencias. Nuestro centro, como ya hemos descrito en trabajos anteriores (Carretero, 2006a, b; Carretero 2007 a, b) pertenece a un área rural muy deprimida de la provincia de Granada donde hay poco interés por el estudio así como escaso hábito lector, sin olvidarnos de la ausencia de un biblioteca municipal. En muchos casos nos encontramos con padres de alumnos prácticamente analfabetos donde sus hijos son los que tienen que leer o escribir por ellos.

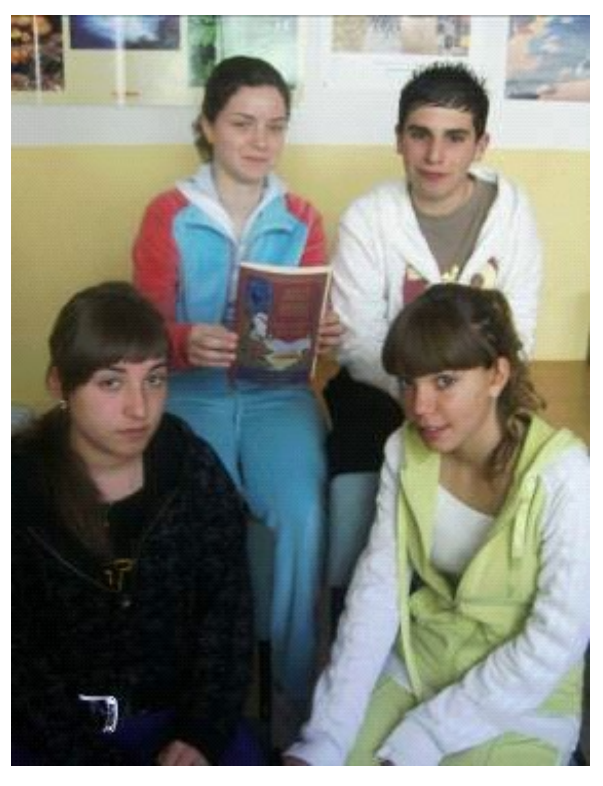

Por otra parte siempre he tenido gran interés en acercar la ciencia y hacerla más accesible a nuestros alumnos (Carretero, 2006a, b; Carretero, 2007a, b), así que pensé que utilizar la ciencia ficción en mis clases Biología sería una buena idea para conseguir este doble objetivo (acercar la ciencia y mejorar la capacidad lectora). Existen experiencias similares a esta en las que los resultados han sido muy interesantes (Guerra, 2004; Guerra, 2005; García Borrás, 2005a, 2005b; García Borrás, 2006; Sardá, Márquez, Sanmartín, 2006; Palacios 2007), así que me decidí a utilizar este recurso.

He trabajado con alumnos de 40 de la ESO pertenecientes a la asignatura de Biología y Geología, con ellos he realizado la lectura de la novela "Viaje al centro de la Tierra" de Jules Verne. La elección de Jules Verne se debe a que todos los de generaciones cercanas a la mía recordamos con entusiasmo las buenas y amenas horas que nos ha hecho pasar durante nuestra adolescencia. Posiblemente son numerosos los científicos que le deben su vocación contribuyendo al bienestar de la humanidad, no habiendo duda de que ha ejercido una vasta influencia educativa y ética (Sierra, 2007). Además este autor no solo es bueno por lo ameno de sus novelas, si no por poder utilizar sus textos para profundizar y afianzar nuestros conocimientos en ciencias. Con este trabajo he querido conseguir que los alumnos desarrollaran la capacidad de relacionar conceptos que aparecen el la lectura con los conocimientos adquiridos previamente en clase. Como bien dice Loscertales y Núñez (1997) 
los alumnos de nuestro sistema educativo son los que configuran el trabajo del profesor, el cual deberá poner en práctica todos los recursos a su alcance para adecuar el proceso de enseñanza-aprendizaje a los intereses, necesidades, y características del alumnado. Por esta razón pensé que acudir a la lectura de textos de ciencia ficción sería una buena forma de hacerlo más asequible.

Esta experiencia ha permitido:

- Comparar los recursos y conocimientos científicos de la época con los existentes en nuestros días, haciendo consciente al alumnado del avance tecnocientífico sufrido.

- Profundizar en conceptos ya explicados previamente en clase.

- Relacionar diferentes ramas de la ciencia, Geología, Matemáticas, Biología, Física.

- Analizar y descubrir algunos errores científicos cometidos por el autor y que dieron lugar a enriquecedores debates y discusiones en clase.

- Manejar un amplio vocabulario científico.

\section{OBJETIVOS Y DESARROLLO DE LA TRAVESÍA}

Después de lo hasta ahora expuesto, creo que las intenciones de la experiencia están bastante claras. Ha sido muy importante prestar atención a no obligarles a hacer nada que no quisieran, intentando atraerlos con los ambientes propios de la ciencia ficción (monstruos, aventuras, batallas etc.) por los que suelen tener cierta preferencia. Se podrían destacar de la experiencia los siguientes objetivos:

- Promover el hábito lector del alumnado. De manera que poco a poco ganen fluidez y comprensión lectora.

- Comenzar a corregir las deficiencias lectoescritoras que presentan.

- Fomentar una lectura crítica, iniciándolos en textos que de alguna u otra forma estuvieran relacionados con la ciencia, incitándolos a informarse y formarse por cuenta propia.

- Desarrollar y aumentar la cultura científica del alumnado de nuestro instituto.

- Hacer más amenas y atractivas las clases de Biología y Geología utilizando como elemento motivador la ciencia ficción.

- Relacionar las diferentes disciplinas científicas entre sí.

- Afianzar, corregir y aumentar conceptos y teorías ya explicados en clase.

- Conocer como la evolución de la ciencia está teniendo lugar a pasos agigantados teniendo cada día más importancia en nuestras vidas.

- Comparar los conocimientos que tiene el autor con los que actualmente tenemos sobre el tema. 
Como ya se ha expuesto previamente, se ha tomado como libro de referencia la novela "Viaje al centro de la Tierra". Dicha elección se ha basado en la necesidad de relacionar la lectura con los contenidos pertenecientes a la asignatura de $4^{\circ}$ de ESO. Estos alumnos habían terminado el curso anterior y comenzado este con los temas de geología. Así que este ha sido un texto bastante adecuado para globalizar los contenidos. Ha servido para afianzar, recordar y profundizar en temas tales como: estructura interna terrestre, corrientes y movimientos de convección, magma, volcanes, tipos de rocas, unidades de medida, campo magnético, dorsales etc. Además ha dado pie al establecimiento de interesantes debates, como el surgido al observar la facilidad con la que los protagonistas de la historia pueden llegar al centro terrestre sin sufrir grandes cambios de presión y de temperatura, estimulando así la capacidad crítica y escéptica de los estudiantes.

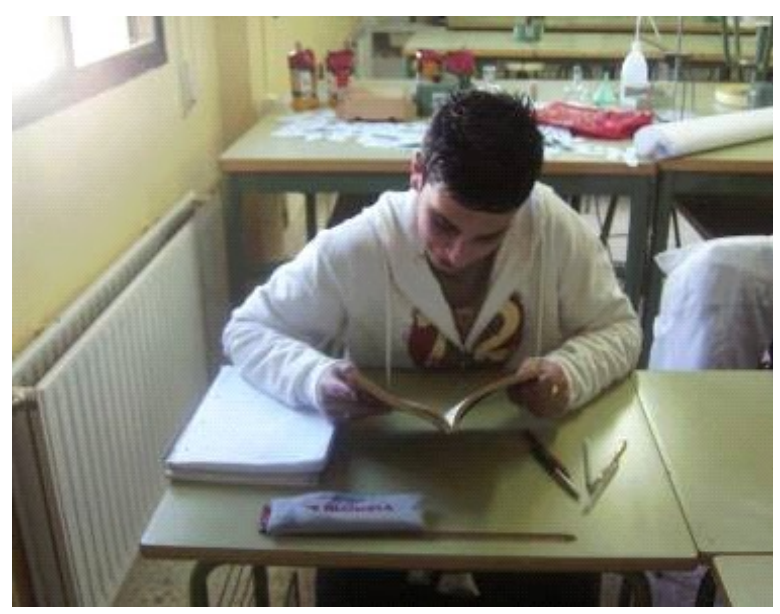

Esta experiencia ha contado con varias partes:

- Lectura completa de la novela. Ha sido el trabajo asignado para vacaciones de Navidad, con la entrega de un resumen de la misma.

- Lecturas de varios fragmentos extraídos del libro.

- Realización de diversas cuestiones relacionadas con dichos fragmentos (Anexo).

- Elaboración, por parte de los alumnos, de su propio relato de ciencia ficción. Un relato corto que les ha permitido desarrollar su habilidad escritora.

Hay diferentes posibilidades de trabajar con las obras literarias bien con el libro completo o con fragmentos, realizando actividades complementarias o con la elaboración de una obra corta de ciencia ficción (Bassa y Freixas 1973; Martín, Gracia, Perera y Pizarro 1985; Guerra 2005; García Borrás 2006). Esta experiencia ha utilizado una mezcla entre todas.

La metodología seguida ha sido similar a la utilizada por otros autores (Loscertales y Núñez, 1997; García Borrás, 2006). Ha sido activa al facilitar el trabajo conjunto entre el alumnado y la profesora, puesto que hemos investigado, descubierto y colaborado entre todos. Por otra parte podemos definirla como dialogante ya que la puesta en común y el debate han sido uno de los pilares de nuestra actividad. Se han entablado discusiones y debates sobre el tema, que han servido de ayuda para que los alumnos fueran objeto de su propio aprendizaje a la vez que se fomentó el trabajo cooperativo entre ellos. Nos ha permitido trabajar de una forma flexible y abierta al ser susceptible de modificarse en función de las necesidades y características del grupo. Ha fomentado el carácter investigador por utilizar el trabajo y el descubrimiento para que el alumnado analice los problemas surgidos, potenciando su autonomía en cuanto a los procesos de análisis, búsqueda de información, reflexión etc. 


\section{CONCLUSIONES: PARADA Y FONDA}

Como ideas finales se pueden indicar que esta experiencia ha supuesto para nosotros una forma de trabajar nueva (aunque ya habíamos intentado algo parecido Carretero, 2006 a).

El alumnado necesita mejorar sus competencias lectoescritoras, lo que se ha intentado corregir utilizando otras formas de trabajar. A la vez ha servido para establecer relaciones entre los conocimientos previos y el texto leído favoreciendo su formación científica y ciudadana. Vivimos en un mundo en el que la ciencia, la tecnología y la sociedad van íntimamente ligadas, ello hace que debamos proporcionar a alumnado la formación adecuada para que sean unos futuros ciudadanos con las herramientas suficientes que les permitan desenvolverse en el mundo al que nos empuja esta revolución científicotecnológica. Una ciencia para todos, así como una alfabetización científico-tecnológica, constituyen los retos actuales de la educación científica en los países educativamente avanzados (Blanco, 2004).

El profesorado debería plantearse la necesidad de incluir como actividad la lectura comprensiva de textos científicos puesto que la lectura es un pilar básico para el aprendizaje de cualquier disciplina y en nuestro caso de las ciencias naturales.

Es necesario emplear nuevos recursos didácticos que hagan a las asignaturas de ciencias más interesantes para el alumnado, haciéndolas más asequibles y no dirigidas a un reducido número de "elegidos".

La ciencia ficción es un recurso de interesante valor para la enseñanza y divulgación de la ciencia, siendo la obra de Jules Verne de gran importancia por su carácter educativo. Este tipo de actividades hacen necesario un tratamiento previo en clase, aclarando, explicando y profundizando sobre el tema a investigar. Es necesario desarrollar diferentes estrategias de compresión lectora en las clases de ciencias, si queremos conseguir un aprendizaje significativo en nuestros alumnos.

Con frecuencia el profesorado nos limitamos a una exposición rutinaria de los contenidos, siendo esta una forma poco atractiva para el alumnado. Debemos intentar cambiar esta actitud y conseguir que la enseñanza de la ciencia sea divertida y pueda entusiasmar a la vez que ayuda al joven a tener una visión global y que pueda descubrir más y mejor el mundo que les rodea. La ciencia ficción nos ayuda a aumentar el interés por las ciencias estimulando la curiosidad del alumnado (Sari, 2003). Fomentamos con ella la capacidad de razonamiento crítico a la vez que desarrollamos el escepticismo en nuestros estudiantes, evitando así que crezcan siendo unas personas irreflexivas.

Hemos comparado conocimientos de épocas diferentes poniendo de manifiesto el avance de la ciencia desde Jules Verne hasta nuestros días. Ello nos ha ayudado a poner en común una serie de ideas que hemos utilizado para debatir diferentes argumentos de una forma razonada y tolerante, fomentando en todo momento un espíritu de libertad y diálogo.

El alumno ha mostrado gran interés no solo por encontrar la respuesta a las cuestiones planteadas, sino también por conocer cual va a ser el resultado de la aventura en la que están inmersos, la lectura. Utilizar tareas creativas puede ayudar afianzar contenidos a la 
vez que mantienen el interés. Algunos de ellos han hecho comentarios como: "ya me había leído antes este libro y no me gustó, ahora lo he comprendido mucho mejor por haber entendido cosas que antes no sabía", "me ha gustado mucho, algunas cosas de las que habla las habíamos visto en clase y me ha facilitado su comprensión"

Es importante que los docentes guiemos y ayudemos a nuestros estudiantes a adquirir formas de trabajar que les aporten nuevos conocimientos y destrezas que contribuyan al desarrollo de sus competencias lectoras (Maturano, Soliveres y Macías, 2002) además de incentivar la motivación y el interés por la ciencia.

Como bien dice Guerra (2004) la literatura y el cine se presentan algo más que interesantes a la hora de intentar modificar la idea de ciencia y del trabajo científico que tiene la ciudadanía en general, ya que, o reflejan acertadamente la visión que queremos transmitir, o bien nos muestran estos tópicos claramente, abriendo la puerta a interesantes debates.

\section{REFERENIAS BIBLIOGRÁFICA}

BACAS, P.; MARTÍN-DÍAZ; PERERA, F.; PIZARRO, A. (1993). Física y Ciencia-Ficción. Madrid: Ediciones Akal.

BLANCO LÓPEZ, A. (2004) Relaciones entre la divulgación científica y la divulgación de la ciencia. Revista Eureka sobre Enseñanza y Divulgación de las Ciencias, 1 (2), 70-86. En línea en: http://www.apac-eureka.org/revista.

BASSA, J.; FREIXAS, R. (1973). El cine de ciencia-ficción. Una aproximación. Editorial

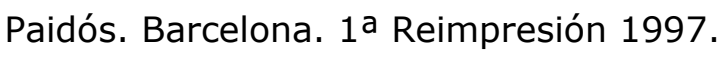

CARRETERO, M.B. (2006). El Quijote: nutrición y salud. Revista Eureka sobre Enseñanza y Divulgación de las Ciencias, 3(1), 134-157. En línea en: http://www.apaceureka.org/revista.

CARRETERO, M.B. (2006b). Celebremos el primer centenario de la teoría de la relatividad conociendo a los científicos y su trabajo. Revista Eureka sobre Enseñanza y Divulgación de las Ciencias, 3(2), 287-299. En línea en: http://www.apaceureka.org/revista.

CARRETERO, M.B. (2007). Wolfgang Amadeus Mozart nos ayuda ha estudiar anatomía. Revista Eureka sobre Enseñanza y Divulgación de las Ciencias, 4(1), 176-188. En línea en: http://www.apac-eureka.org/revista.

CARRETERO, M.B. (2007b). Aprendamos a conocer y a respetar el medio que nos rodea de la mano de Leonardo da Vinci. Revista Eureka sobre Enseñanza y Divulgación de las Ciencias, 4(2), 325-338. En línea en: http://www.apac-eureka.org/revista.

FERNÁNDEZ, I.; GIL, D.; CARRASCOSA, J.; CACHAPUZ, A.; PRAIA, J. (2002). Visiones deformadas de la Ciencia transmitidas por la enseñanza. Enseñanza de las

Ciencias, 20(3), 477-488. En línea:

http://www.bib.uab.es/pub/ensenanzadelasciencias/02124521v20n3p477.pdf 
GARCÍA BORRÁS F. J. (2006) Cuando los mundos chocan. Revista Eureka sobre Enseñanza y Divulgación de las Ciencias, 3(2), 268-286. En línea: http://www.apaceureka.org/revista

GALLEGO TORRES, A.P. (2007) Imagen popular de la ciencia transmitida por los cómics. Revista Eureka sobre Enseñanza y Divulgación de las Ciencias, 4(1) 141-151 En línea: http://www.apac-eureka.org/revista.

GARCÍA BORRÁS, F.J. (2005a). La serie C.S.I. como metáfora de algunas facetas del trabajo científico. Revista Eureka sobre Enseñanza y Divulgación de las Ciencias, 2(3), 374-387. En línea: http://www.apac-eureka.org/revista.

GARCÍA BORRÁS, F.J. (2005b). Star Trek: Un viaje a las leyes de la dinámica. Revista Eureka sobre Enseñanza y Divulgación de las Ciencias, 2(1), 79-90. En línea: http:// www.apac-eureka.org/revista.

GUERRA RETAMOSA, C. (2004). Laboratorio y batas blancas en el cine. Revista Eureka sobre Enseñanza y Divulgación de las Ciencias, 1(1), 52-63. En línea en: http://www.apac-eureka.org/revista

GUERRA RETAMOSA, C. (2005). Náufragos, amantes y aventureros en el aula. Revista Eureka sobre Enseñanza y Divulgación de las Ciencias, 2(2), 173-182. En línea: http://www.apac-eureka.org/revista.

LOSCERTALES ABRIL, F.; NÚÑEZ DOMÍNGUEZ, T. (1997) Técnicas de trabajo en el aula con prensa Edutec 97: Creación de materiales para la innovación educativa con nuevas tecnologías. III Congreso de Nuevas Tecnologías de la Información para la Educación En linea:http://www.ieev.uma.es/edutec97/edu97 ta/taller03.htm.

MÁRQUEZ, CONXITA Y PRAT, ÀNGELS. (2005) Leer en clase de ciencias. Enseñanza de las ciencias, 23(3), 431-440

MARTÍN, M.J.; GARCÍA, J.P.; PERERA, F.; PIZARRO, A. (1985). Ciencia y fantasía en el aula. Cuadernos de Pedagogía, 162, 40-43.

MATURANO, C.I, SOLIVERES, M.A. y MACÍAS, A. (2002) Estrategias cognitivas y metacognitivas en la comprensión de un texto de ciencias. Enseñanza de las ciencias, 20 (3) 415-425

MATURANO, C; MAZZITTELI, C.; MACÍAS A. (2006). ¿Cómo los estudiantes regulan la comprensión cuando leen un texto instructivo con dificultades? Revista electrónica de enseñanza de las ciencias, 5(2), 235-246.

PALACIOS, S.L. (2007). El cine y la literatura de ciencia ficción como herramientas didácticas en la enseñanza de la física: una experiencia en el aula. Revista Eureka sobre Enseñanza y Divulgación de las Ciencias, 4(1), 106-122. En línea: http://www.apac-eureka.org/revista. 
SARDÁ, A.; MÁRQUEZ, C; SANMARTÍN, N. (2006) Como promover distintos niveles de lectura de los textos de ciencias. Revista electrónica de enseñanza de las ciencias, $5(2), 290-303$

SARI, M. (2003). Las ciencias para todos... Revista de investigación europea 37 En línea en: http://europa.eu.int/comm/research/rtdinfo/37/letters es.html.

SIERRA CUARTAS, C.E. (2007) Fortalezas epistemológicas y axiológicas de la ciencia ficción: un Potosí pedagógico mal aprovechado en la enseñanza y divulgación de las ciencias. Revista Eureka sobre Enseñanza y Divulgación de las Ciencias, 4(1), 87-105 En línea: http://www.apac-eureka.org/revista. 


\footnotetext{
ANEXO

A continuación tienes una serie de preguntas relacionadas con el libro que hemos leído responde a ellas los más explícitamente que puedas.

¿Quien se supone que escribe el libro?.

Quienes son los personajes, como se llaman, a que se dedican etc. Haz una breve descripción de cada uno de ellos.

¿Cómo se describe en el libro al profesor Liderbrock?.

¿Qué quiere decir el autor con la frase referida al profesor Linderbrock "enseñaba subjetivamente"?.

¿Qué es la mineralogía?.

"El calor aumenta 10 cada 79 pies" ¿A que nos referimos con la palabra pie?, ¿a cuantos cm. corresponde?, ¿coincide con los datos que hoy sabemos?, ¿como llamamos a esta característica de la Tierra?.

"El radio terrestre mide 1500 leguas" ¿Qué es una legua?, ¿a cuanto corresponde?, ¿coincide con la realidad?.

Según Axel el límite de la corteza terrestre se encuentra "a diez leguas del límite de corteza terrestre" ¿es cierto?.

¿Cómo explica que la temperatura en el interior terrestre no sea tan alta?.

¿Qué son las calendas?.

¿Qué herramientas e instrumentos llevan en el viaje? ¿que utilidad tienen?.

¿Por qué en Islandia no se pone el Sol durante los meses de junio y julio?.

¿Qué es un fiordo?.

¿Cómo se describe en el texto la formación de Islandia? Relaciónalo con los contenidos que conoces.

¿Crees que el autor ha cometido un fallo cuando dice que a las once de la noche era oscuridad completa? Razónalo.

Entran por el cráter de un volcán ¿crees que es posible? Razónalo.

"tendremos cada vez más aire, al contrario que los astronautas" ¿Qué quiere decir con esta expresión? ¿Crees que es cierto?.

"Solo había aumentado la temperatura $4^{\circ} \mathrm{C}$, el descenso era horizontal" ¿Cómo explican que el ascenso no sea mayor?.

¿En que pruebas se basan para datar los estratos en los que se encuentran?.

¿Cómo explican la formación de las minas de carbón?.

¿Qué es un geiser? ¿cómo se forma?.
} 
¿Por qué suponen que la intensidad de la gravedad disminuirá con forme desciendan?.

¿En que se basan para calcular la distancia a la que están de la superficie? Haz el cálculo en metros.

¿Por qué razón son ciegos los peces que encuentran en el mar de Linderbrock?.

¿Crees que con los conocimientos científicos que tenemos, la brújula marca correctamente la dirección?.

¿A que medida equivale una toesa y una legua?.

¿En que capa terrestre se encuentran cuando son arrastrados por la corriente de material caliente $y$ ven este aumento de temperatura?.

¿Cómo explicarías la existencia de la corriente que los arrastra hasta la superficie? ¿Qué nombre recibe?.

¿Crees que es posible que pudieran salir por el volcán? Con los conocimientos que ya tienes, da una respuesta razonada.

Explica que parte de realidad y de fantasía tiene el libro. ¿Crees posible esta experiencia en la realidad?.

Haz un resumen.

Comenta tus impresiones tras la lectura.

Escribe tu propio cuento de ciencia ficción. 


\section{JOURNEY TO THE CENTER OF BOOK-READING WITH JULES VERNE}

\section{SUMMARY}

This work has been focused on reading science-fiction books at sciences classes. The main objective of this experience was to make students relate concepts found in their reading to concepts learned in class. By choosing science fiction books we wanted students to be interested in reading and at the same time we wanted them to ask their doubts to the teacher. As mentioned by Loscertales and Nuñez (1997), the students give shape to the teacher's work; and the teacher uses all the possible tools to adapt the teaching-learning process to their necessities. We show a way to get science close to Biology students. Science fiction books help us to fulfil this objective. They stimulate their curiosity for sciences issues and improve their reading abilities. To achieve this we chose the novel "Journey to the Centre of the Earth" by Jules Verne.

Keywords: Science learning; science fiction books; reading encouraging; didactic resource; scientific methodology; scientific behaviour. 